\title{
Caracterización del modelo HEC-HMS en la cuenca de río Arga en Pamplona y su aplicación a cinco avenidas significativas
}

\section{Characterisation of the HEC-HMS model to the river Arga's watershed in Pamplona and its application to five significant floods}

Fecha de entrega: 21 de febrero 2012

Fecha de aceptación: 14 de junio 2012

\section{J. Javier López ${ }^{1}$, Miguel González ${ }^{1}$, Anna Scaini ${ }^{1}$, Mikel Goñi $^{1}$, José V. Valdenebro ${ }^{1,2}$ y Faustino N. Gimena ${ }^{1}$}

\footnotetext{
${ }^{1}$ Departamento de Proyectos e Ingeniería Rural, Universidad Pública de Navarra, Campus Arrosadía, 31006 Pamplona, Navarra, España.jjlr@unavarra.es, mikel.goni@unavarra.es, faustino@unavarra.es

${ }^{2}$ Área de Proyectos Estratégicos, Ayuntamiento de Pamplona, Plaza Consistorial s/n, 31001 Pamplona, Navarra, España, j.valdenebro@pamplona.es
}

Pamplona es una ciudad que es atravesada por el río Arga a lo largo de una llanura aluvial, que es susceptible de inundaciones cuando se producen avenidas de cierta magnitud. Ante esta situación es importante contar con un modelo hidrológico que permita simular los caudales del río que atraviesa el núcleo urbano, a partir de los datos de los distintos pluviómetros existentes en la cuenca, y que sirva para alimentar a modelos hidráulicos que permitan definir las zonas inundables asociadas a distintos niveles de probabilidad. Con esta finalidad, se ha montado y caracterizado el modelo HEC-HMS de la cuenca del rio Arga en Pamplona, y posteriormente, se ha aplicado a las cinco avenidas más significativas de los últimos años, de las que se disponen de los mínimos datos de caudal y precipitación necesarios. HEC-HMS es un modelo lluviaescorrentía que se basa en estructurar la cuenca origen en subcuencas asociadas a los cauces de la red fluvial. El flujo base en los hidrogramas observados se ha estimado mediante el filtro de Eckhardt. Después de realizar un análisis de sensibilidad de los parámetros inciertos del modelo, en el que se ha observado que el más sensible es el CN, se ha aplicado el modelo con los datos de las series de precipitación de las estaciones automáticas, y con los datos de las automáticas más las manuales, en este segundo caso los resultados han mejorado significativamente obteniéndose resultados satisfactorios.

Palabras clave: HEC-HMS, simulación de avenidas, modelos lluvia-escorrentía
Pamplona is a city that is crossed by the river Arga along a floodplain, which is susceptible to flooding when there are floods of certain magnitude. In this situation it is important to have a hydrological model able to simulate the flows that runs through the city centre, from the data of different gauges in the watershed, which serve to feed hydraulic models that permits to define the flooding susceptible areas associated to different levels of probability. With this end, the HEC-HMS model of river Arga's watershed in Pamplona, has been assembled and characterised, and subsequently applied to the five most significant floods in recent years, from which the minimum required data of flow and precipitation is available. HEC-HMS is a rainfallrunoff model that consists of structuring the source watershed into sub-watersheds associated to the river network. The base flow in the observed hydrographs was estimated by the Eckhardt filter. After performing a sensitivity analysis of uncertain parameters of the model (in which it has been observed that $C N$ is the most sensitive) the model has been applied with data from the precipitation series of the automatic stations and data from automatic plus manual stations; in the latter case the results have improved significantly, obtaining satisfactory results.

Keywords: HEC-HMS, flood simulation, rainfall-runoff models 


\section{Introducción}

Los ríos Arga y Ulzama que nacen en la zona montañosa al norte de Navarra, España, se caracterizan por ser ríos de montaña que discurren por valles estrechos y con pendientes acusadas. Al llegar a la Cuenca de Pamplona, donde se ubican diversas poblaciones en torno a la capital Navarra (Pamplona), ambos ríos se unen duplicando su caudal, reducen considerablemente su pendiente adoptando una forma meandriforme, y atraviesan extensas plataformas inundables ocupadas por desarrollos urbanísticos con usos residenciales, dotacionales e industriales (Figura 1). Estas zonas en momentos de riadas o avenidas son susceptibles de ser anegadas (Figura 2), como se demuestra en una revisión de hemeroteca llevada a cabo por Iglesias (2008), en la que se pone de manifiesto que en las últimas tres décadas se han contabilizado más de una treintena de fenómenos tormentosos que han producido daños o problemas asociados a inundaciones en la cuenca de Pamplona. Por otra parte, las avenidas más importantes de los últimos 15 años se han producido en febrero de 2003, marzo de 2006 y febrero de 2009, cuyos valores de los periodos de retorno, estimados por el Gobierno de Navarra, se cifran en 10,5 y entre 10 y 15 años respectivamente, lo que resulta coherente con los resultados de la revisión citada. Aunque el río Arga es el río más caudaloso que atraviesa Pamplona, no es el único que lo hace y que tiene afecciones sobre la ciudad, por el sur el río Elorz y su afluente el Sadar cruzan a través de algunas poblaciones y barrios de Pamplona (Figura 1), uniéndose al río Arga poco después de haberla atravesado.

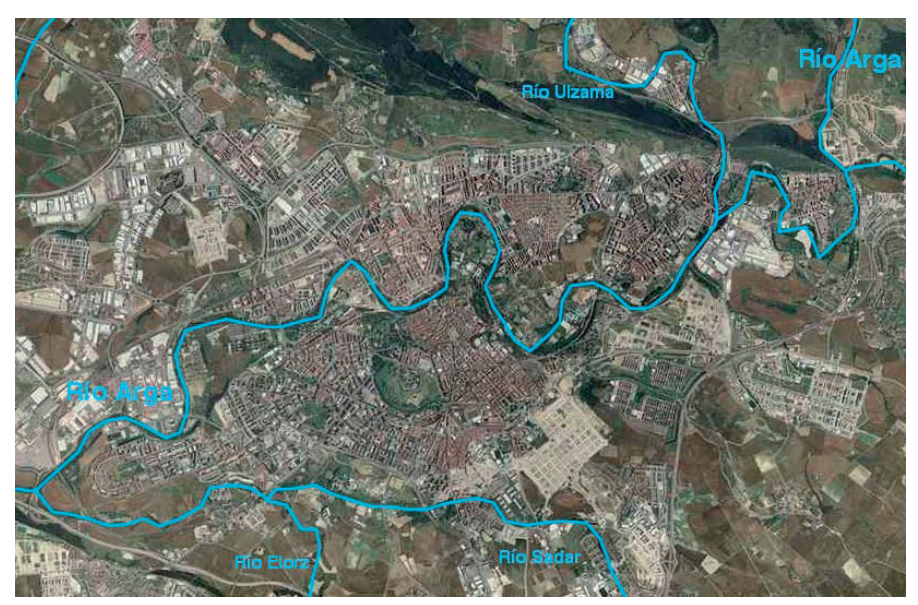

Figura 1: Ortofoto de la cuenca de Pamplona con la red hidrográfica marcada (SITNA)
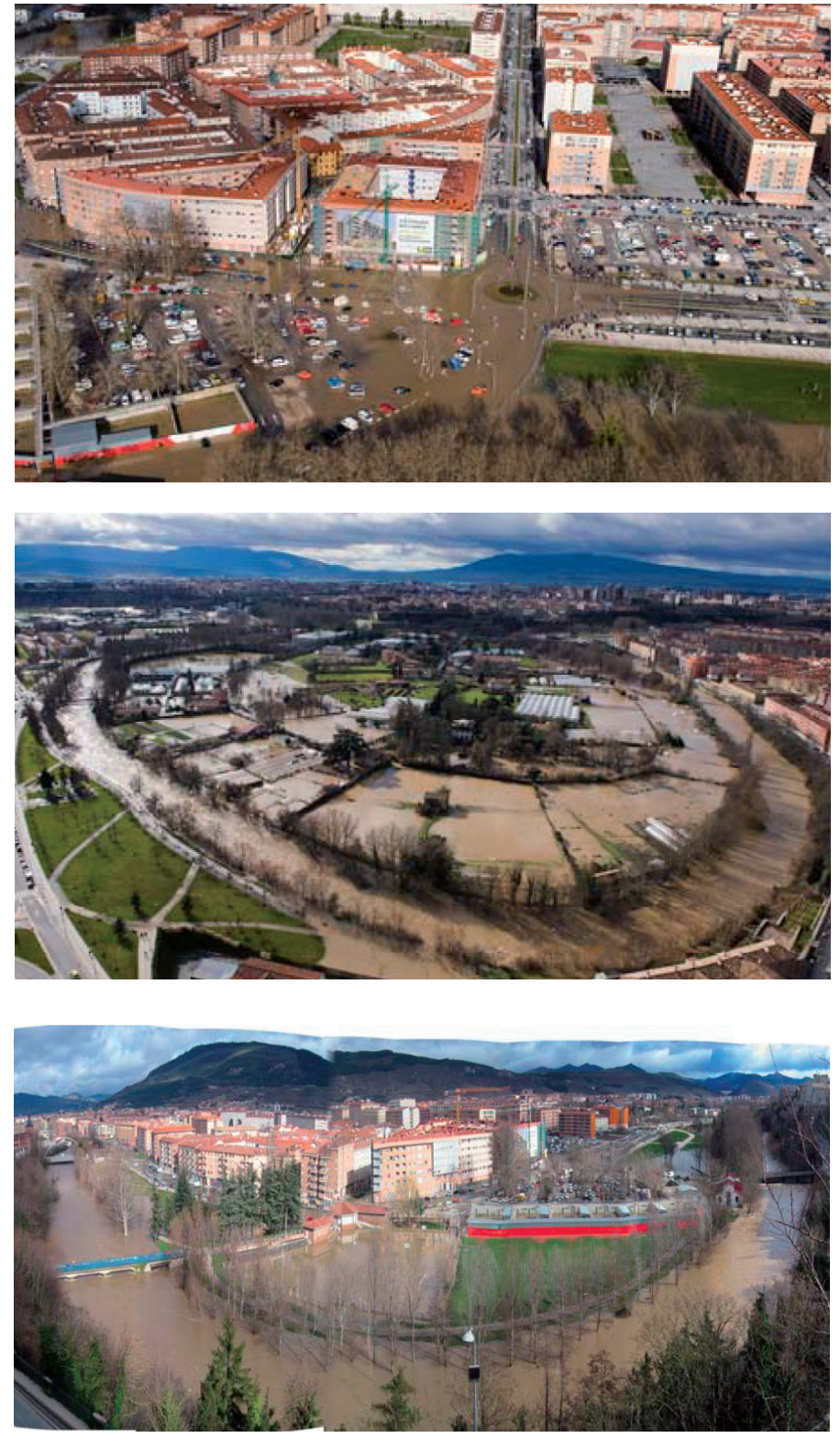

Figura 2: Fotos de las inundaciones del 12 de febrero de 2009 en Pamplona

Podemos decir que Pamplona, con unos 200.000 habitantes, se asienta fundamentalmente sobre dos ámbitos: uno la Meseta, muy elevada con respecto al nivel del río; y otro el aluvial del río en el que existen riesgos de inundación fundamentalmente en las zonas más próximas al río. El Ayuntamiento de Pamplona a través de los Planes de Ordenación Urbanísticos ha ido buscando devolver al río el terreno que le pertenece. De esta forma, en el Plan General de 1984, Pamplona se plantea la recuperación de este hábitat natural como un objetivo prioritario, integrando el entorno fluvial del río Arga en la ciudad como un gran Parque Fluvial, no solo como un espacio público de ocio 
sino como motor de un urbanismo racional y sostenible.

Posteriormente, en el Plan Municipal aprobado en el 2002 (Ayuntamiento de Pamplona, 2003) no solamente se engloba este entorno fluvial sino que lo completa con la integración de los parques fluviales de los ríos Elorz y Sadar (Figura 1). Los objetivos de esta planificación se han podido satisfacer gracias a la implementación de un Plan Integral de actuación en los Ríos de Pamplona, cofinanciado por Europa al $80 \%$, que se ha acometido en tres fases y en estos momentos se encuentra en la tercera.

Todo este esfuerzo de planificación y actuación se ha traducido en una minimización de los daños asociados a inundaciones, ya que, a pesar de la frecuencia de avenidas y de la gran extensión de suelo urbano susceptible, en la mayor parte de estas situaciones lo que se ve afectado en general son parques y, con menor frecuencia para las de mayor magnitud, algún aparcamiento y algunas viviendas y dotaciones deportivas próximas al río.

A partir del 2007, con la aprobación de la Directiva 2007/60/CE (CEE, 2007) relativa a la evaluación y gestión de los riesgos de inundación, se establece en Europa un marco legislativo para la reducción de los daños causados por inundaciones, ya sea a personas, al medio ambiente, al patrimonio cultural, o a la actividad económica. Esta directiva obliga a las Administraciones de los Estados miembros a: realizar una evaluación preliminar del riesgo; preparar mapas de peligrosidad y de riesgo; y diseñar planes de gestión del riesgo de inundación. En España su transposición se ha realizado mediante Real Decreto 903/2010, de 9 de julio, de Evaluación y Gestión de Riesgos de Inundación.

Ante estas exigencias, y como paso previo a la simulación hidráulica que permita estimar las manchas o zonas inundables, es importante poner a punto una herramienta que permita determinar los caudales que circularían por los distintos tramos de ríos que atraviesan el núcleo urbano ante diferentes hipótesis de diseño o distintos escenarios de cambio global. Esta herramienta es un modelo hidrológico Lluvia-Escorrentía que, a partir de los datos de los distintos pluviómetros con influencia en una cuenca, es capaz de determinar los caudales que circulan por los distintos tramos de río. En este caso el modelo seleccionado ha sido el HEC-HMS, un modelo de libre acceso y disponibilidad cuya aplicación está muy extendida en el ejercicio profesional. Entre otras aplicaciones, HEC-HMS se ha utilizado para evaluar los efectos en los hidrogramas de posibles cambios climáticos, de cambios de uso del terreno, de incendios (Cunderlik y Simonovic, 2004; Kang y Ramírez, 2007; McColl y Aggett, 2007; Yusop et al., 2007; Cydzik y Hogue, 2009; McLin et al., 2001); para el diseño y gestión de estructuras hidráulicas (Emerson et al., 2005); para la estimación de recursos hídricos, en su forma continua (Al-Abed et al., 2005; Chu y Steinman, 2009); y para delimitación de zonas inundables (Treviño y Ortiz, 2004; Knebla et al., 2005).

El objetivo del presente trabajo es discutir el uso del modelo HEC-HMS para la estimación de los caudales del río Arga, asociados a distintos periodos de retorno, a su paso por Pamplona.

El modelo desarrollado en HEC-HMS permitirá evaluar el efecto de posibles cambios globales (cambio climático, cambios de uso, entre otros) en la magnitud de las avenidas del mismo.

Además de esta sección introductoria, el trabajo se estructura con un apartado de Materiales y métodos, otro para Resultados y discusión, y el de Conclusiones. En la sección de Materiales y métodos se describen: la cuenca objeto de estudio; el modelo HEC-HMS; y los eventos y el método de extracción del flujo base. En el de Resultados y discusión se presenta, en primer lugar, un epígrafe dedicado a la implementación del modelo en donde se especifican los valores de los parámetros asignados en este caso concreto; en segundo lugar se presenta un análisis de sensibilidad de los parámetros más significativos del modelo; y, por último, el análisis de los resultados de la aplicación del modelo a los eventos seleccionados. Finalmente se exponen las conclusiones del trabajo.

\section{Materiales y métodos}

Se han seleccionado cinco episodios de avenida, los más importantes en magnitud y en efectos, que se han producido en el periodo en el que se disponen datos de precipitación de diez minutos en las Estaciones Meteorológicas Automáticas EMA, periodo relativamente corto comprendido desde el año 2000 hasta la actualidad. Para paliar la escasez de EMAs en la zona montañosa de 
cabecera, se ha evaluado la incorporación de los datos diarios de precipitación de las Estaciones Meteorológicas Manuales EMM, con mucha mayor presencia en esa zona, a los que se ha aplicado la distribución temporal de las estaciones automáticas.

Para ello, se ha contado con los registros de diez minutos de caudal de la estación de aforo de Arazuri perteneciente a la Confederación Hidrográfica del Ebro CHE. Así mismo, se ha dispuesto de los registros de precipitación diaria y cada 10 minutos, de las estaciones manuales EMM y automáticas EMA respectivamente, repartidas por toda la superficie de la cuenca. Por otra parte, se han estimado los parámetros de los componentes del modelo HEC-HMS para la cuenca de Arga y para cada uno de los eventos.

\section{Descripción de la cuenca}

Navarra, con una superficie aproximada de $10.391 \mathrm{~km}^{2}$, se sitúa al norte de España y al suroeste de la cordillera pirenaica (Figura 3). Su capital, Pamplona, localizada más o menos en el centro de Navarra, está rodeada de distintas cadenas montañosas formando lo que se denomina la Cuenca de Pamplona. Aunque de vertiente mediterránea, la cercanía a las divisorias atlánticas y la relativamente escasa altitud de las mismas, proporciona a toda la zona una situación de transición climática y biogeográfica muy fuerte.

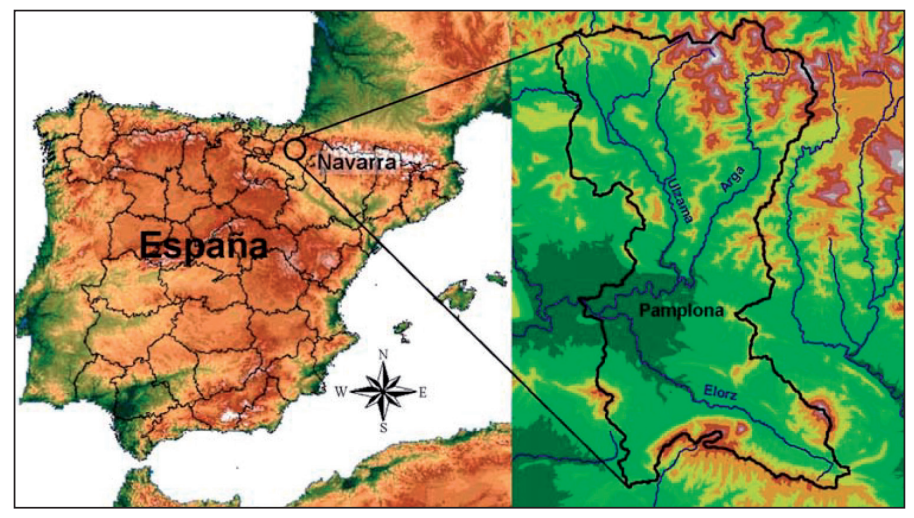

Figura 3: Localización y delimitación de la cuenca del Arga una vez atravesado Pamplona

Como se puede ver en la Figura 3, el río Arga nace en los montes de Quinto Real, en la vertiente meridional al norte de Navarra (España). Poco después toma dirección sur, a través del Valle de Esteríbar, hasta llegar a la entrada de la Cuenca de Pamplona, en donde recibe al río Ulzama en el municipio de Huarte, afluente de gran importancia que duplica el caudal del Arga. En Huarte, el río Arga presenta un caudal medio anual de $7.18 \mathrm{~m}^{3} / \mathrm{s}$ y justo a la entrada de la cuenca recibe al citado río Ulzama, que procede de los montes de Ulzama-Velate y que, con su caudal medio anual de $8.48 \mathrm{~m}^{3} / \mathrm{s}$, hace que se duplique el caudal original del río Arga.

Ambos ríos, hasta llegar a la entrada de la Cuenca de Pamplona, se caracterizan por ser ríos de montaña, con pendientes acusadas que discurren por valles estrechos. Al entrar en la cuenca, el río Arga adquiere un recorrido meandriforme, recibiendo al río Elorz antes de dejar Pamplona y llegar al punto en el que se ubica la estación de aforo de Arazuri, perteneciente a la Confederación Hidrográfica del Ebro. La superficie de la cuenca que drena en este punto, estación de aforo de Arazuri, es de 803.49 $\mathrm{km}^{2}$ y la red fluvial queda definida fundamentalmente por los ríos Arga, Ulzama y Elorz.

En Pamplona la vega presenta una anchura de 500 a 1500 $\mathrm{m}$ y su régimen hidrológico manifiesta crecidas de gran magnitud, que pueden afectar a una amplia extensión de terreno.

Como consecuencia de todas estas características, el Arga debería considerarse como un río de gran dinamismo, con fenómenos de erosión en la cara externa de los meandros y ripas, sedimentación y formación de depósitos en la cara interna de los mismos, así como tendencia natural a cambiar el trazado en la llanura aluvial. Este dinamismo natural se encuentra muy alterado y casi totalmente eliminado por la presencia de numerosas infraestructuras, construcciones y aprovechamientos por lo que se puede considerar que su curso está fijado.

A nivel geológico, la cabecera de la cuenca del río Arga se asienta en los terrenos Paleozoicos, aguas abajo aparecen substratos de caliza dura y flish. La parte media de la cuenca está formada por margas, además de arcillas y areniscas. La cuenca del río Ulzama también nace en el macizo Paleozoico, con composición muy variada en su parte alta. Aguas abajo, entra en terrenos Eocénicos de flish intercalados con calizas arcillosas, que en su tramo final enlazan con las margas. El río Elorz queda inmerso en las margas Eocénicas que ocupan el fondo de la cuenca, entre las areniscas de la Sierra de Tajonar y los niveles calizos de la Sierra de Aláiz (Figura 4a). En cuanto a la vegetación presente en la cuenca, tal como se muestra en 
la Figura 4b, al norte de la cuenca del Arga, en las zonas altas, predominan los hayedos. Aguas abajo, la cuenca queda colonizada por el bosque de roble pubescente con pino albar, siempre acompañado por el boj como especie más abundante. En la llanura de inundación aparecen los terrenos de cultivo. La cuenca del Ulzama, en su cabecera, es de características similares a la del Arga, aguas abajo aparecen amplias praderas en el fondo del valle aprovechadas por una rica cabaña ganadera. La cuenca del río Elorz se compone fundamentalmente de cultivos herbáceos y cereales, además de repoblación de pino de Austria. En las sierras aparecen hayedos en las partes altas, colonizados por robledales con el boj como especie acompañante.

El clima se puede clasificar, según el método de Papadakis, como "Meth: Mediterráneo templado" (http://meteo. navarra.es/win/papadakis.cfm), cuya fórmula climática es AvMME (Av: invierno Avena; M: verano Maíz; ME: Mediterráneo húmedo). La temperatura media anual varía entre $10^{\circ} \mathrm{C}$ en las zonas montañosas del norte de la cuenca y $\operatorname{los} 12.5^{\circ} \mathrm{C}$ en la zona más baja de la cuenca, Pamplona, con unas temperaturas más altas en los meses de julio y agosto y menores de diciembre a febrero. La evapotranspiración potencial ETP media, estimada por Thornthwaite, no alcanza valores superiores a $700 \mathrm{~mm} / \mathrm{año}$, siendo inferior a 650 en algunas zonas de las cabeceras y próximo a los 680 en Pamplona. El régimen de precipitaciones es estacional, siendo las estaciones con mayor pluviometría otoño y primavera, y julio y agosto los meses con el mínimo pluviométrico. La precipitación media de la cuenca del río Arga para el periodo 1940-2000 es 972 mm/año, variando entre unos $1600 \mathrm{~mm} / \mathrm{año}$ en zonas altas de la cuenca y unos 750 mm/año en Pamplona. El régimen de aportaciones también es estacional y variable, la aportación media de toda la cuenca del río Arga es de $1559 \mathrm{Hm}^{3} /$ año, siendo el año de mayor aportación 1914/15 con casi $3000 \mathrm{Hm}^{3}$ y el de menor el 2000/2001 con $511 \mathrm{Hm}^{3}$. Las aportaciones medias anuales de las tres subcuencas que drenan a Pamplona son: Alto Arga hasta el Ulzama $188 \mathrm{Hm}^{3}$, Ulzama $219 \mathrm{Hm}^{3}$ y Elorz $70 \mathrm{Hm}^{3}$.

\section{Descripción del modelo hidrológico HEC- HMS}

El modelo HEC-HMS (Hydrologic Engineering Center-

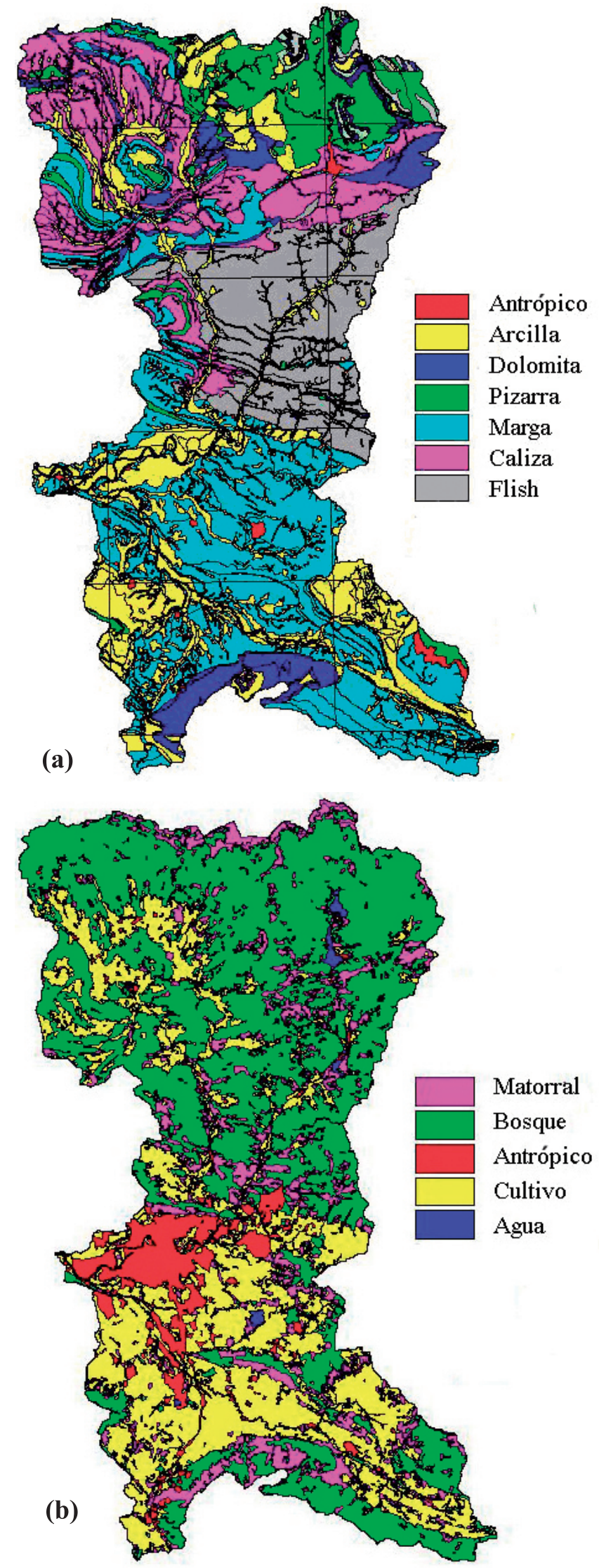

Figura 4: a) Mapa Geológico y b) mapa de usos de la cuenca del río Arga hasta la estación de aforo de Arazuri 
Hydrologic Modeling System) es un modelo lluviaescorrentía, desarrollado por el Hydrologic Engineering Center HEC del U.S. Army Corps of Engineers USACE, que está diseñado para simular el hidrograma de escorrentía que se produce en un determinado punto de la red fluvial como consecuencia de un episodio de lluvia. El antecedente de este modelo, el HEC-1, nació como un modelo de eventos y ha sido considerado por muchos como el modelo más versátil (Bedient y Huber, 1992) y probablemente el más ampliamente utilizado en este tipo de caracterizaciones hidrológicas de avenidas. En este trabajo se ha utilizado el HEC-HMS 3.5.

La simulación de la red de drenaje de una cuenca constituye la base del modelo. Todas las demás opciones están desarrolladas sobre la capacidad de cálculo de hidrogramas en cualquier punto de la cuenca (Viessman y Lewis, 1996). Los componentes del modelo funcionan basados en relaciones matemáticas simples que tratan de representar los procesos que intervienen en la generación y circulación de los hidrogramas de escorrentía, por ejemplo, pérdidas, transformación del exceso de lluvia en caudal de salida de la cuenca, adición del flujo base y circulación del hidrograma por el cauce. El punto de partida, por lo tanto, es la representación distribuida de la cuenca basada en una red ramificada de sistemas hidrológicos-hidráulicos (subcuencas-embalses) conectados por sistemas hidráulicos (cauces).

Cada sistema se caracteriza fundamentalmente por dos elementos: una serie de parámetros que especifiquen las características particulares del mismo, y unas relaciones matemáticas que rigen su comportamiento.

La entrada al modelo es la precipitación evaluada para cada subcuenca, que se hace mediante el método de los Polígonos de Thiessen (Chow et al., 1989), a partir de los registros de precipitación de las estaciones pluviométricas con influencia en cada subcuenca. Por lo tanto, los valores de precipitación del hietograma en cada subcuenca, $P_{s b}$, y para cada intervalo de tiempo, $i$, que se suponen uniformes en toda la superficie de cada subcuenca, se determinan como una media ponderada de las precipitaciones, $P_{e m}$, de las distintas estaciones meteorológicas, $j$, con influencia en la subcuenca, según la ecuación (1):

$$
P_{s b}(i)=\frac{\sum_{j=1}^{n} P_{e m}(i, j) w(j)}{\sum_{j=1}^{n} w(j)}
$$

Donde $P_{s b}(i)$ es la precipitación media uniforme sobre una subcuenca en el intervalo $i, P_{e m}(i, j)$ es la precipitación registrada en el intervalo $i$ y estación $j, w(j)$ es el área de influencia de la estación $j$ en la subcuenca, $n$ es el número de estaciones meteorológicas con influencia en la subcuenca.

La precipitación efectiva es la fracción de la precipitación total que se transforma en escorrentía directa, el resto de la precipitación es considerada como pérdidas o abstracciones.

Para el cálculo de estas pérdidas, HEC-HMS cuenta con diferentes métodos. En este caso, se ha elegido el método del Servicio de Conservación de Suelos SCS, o también llamado del número de curva $C N$, por contar con una información del uso y tipo de suelo digitalizada de calidad. Este método fue desarrollado por el SCS del Departamento de Agricultura de los EE.UU., USDA, para estimar las pérdidas (o abstracciones) en un evento de lluvia o aguacero (Mockus, 1969), y hoy en día es uno de los más utilizados en el ámbito profesional. En este método la altura de lluvia efectiva es función del volumen de precipitación total y de un parámetro de pérdidas denominado número de curva $\mathrm{CN}$. El número de curva varía en el rango de 0 a 100 y depende de factores que influyen en la generación de escorrentía en la cuenca: tipo hidrológico del suelo (Grupo hidrológico-Capacidad de drenaje); uso y manejo del terreno; condición superficial del suelo; y condición de humedad antecedente.

Esta metodología establece que la escorrentía superficial directa comienza después de que se alcance un cierto valor de lluvia acumulada, $I_{a}$, denominada pérdida (o abstracción) inicial. En base a datos de numerosas cuencas pequeñas experimentales instaladas a lo largo de los EE.UU., se llegó a la expresión $I_{a}=0.2 \mathrm{~S}$, donde el coeficiente 0.2 es el valor de un parámetro de ajuste, recomendado por el SCS como estándar del coeficiente de pérdidas iniciales en base a ese gran número de datos experimentales. Los estudios realizados por el SCS permitieron relacionar la retención 
máxima potencial, $S$, con un parámetro de referencia empírico y adimensional que se denomina número de curva, $C N$, cuya expresión en mm es:

$$
S=254\left(\frac{100}{C N}-1\right)
$$

\section{Circulación de la lluvia efectiva a través de la cuenca}

Este proceso hace referencia a la generación del hidrograma en el punto de drenaje de una cuenca como consecuencia de la circulación de la lluvia efectiva a través de toda la superficie de la cuenca, componente que representa la escorrentía superficial directa. HEC-HMS, al igual que para los demás componentes del modelo, dispone de diferentes métodos para determinar este hidrograma de escorrentía directa. En este trabajo se ha seleccionado la técnica del hidrograma unitario HU y, dentro de ésta, se ha elegido el HU Adimensional del SCS por ser un método sencillo ampliamente aplicado en multitud de casos. Este HU se desarrolló en el SCS, en la década de los 50, también en base a observaciones de numerosas cuencas agrícolas repartidas a lo largo de toda la geografía de los EE.UU. Las variables están adimensionalizadas, haciendo el mismo de ámbito universal, de forma que el caudal se representa como $q / Q_{p}$, siendo $Q_{p}$ el caudal máximo o punta generado en la cuenca objeto de estudio; y el tiempo como $t / T_{p}$, donde $T_{p}$ es el tiempo para el caudal máximo. Esta metodología establece que (Chow et al., 1989):

$$
Q_{p}=\frac{2.08 A}{T_{p}}
$$

Donde $Q_{p}$ es el caudal punta en $\mathrm{m}^{3} / \mathrm{s}$ por $\mathrm{cm}$ de lluvia efectiva; $A$ es la superficie de la cuenca en $\mathrm{km}^{2}$; y $T_{p}$ es el tiempo al máximo en horas, cuyo valor es,

$$
T_{p}=\frac{T_{y}}{2}+T_{l}
$$

Siendo $T_{y}$ la duración del pulso de lluvia y $T_{l}$ el tiempo de desfase de la cuenca, definido como el tiempo comprendido entre el centro de gravedad del pulso de lluvia y el instante del caudal máximo, que se determina mediante la expresión (5) (Ponce, 1989),

$$
T_{l}=\frac{L^{0,8}(2.540-22,86 C N)^{0,7}}{14.104 C N^{0,7} I^{0,5}}
$$

En la que $T_{l}$ viene dado en horas, $L$ es la longitud hidráulica en $\mathrm{m}$ (longitud medida a lo largo del cauce principal hasta la divisoria de la cuenca), $I$ es la pendiente media de la cuenca en $\mathrm{m} / \mathrm{m}$, y $C N$ es el valor del número de curva medio de la cuenca.

\section{Circulación de los hidrogramas a lo largo de los cauces}

La circulación de avenidas o hidrogramas hace referencia a como se propagan las avenidas a través de la red de cauces. De los diferentes métodos que HEC-HMS tiene implementados para circular los hidrogramas generados en las subcuencas a través de los cauces se ha elegido el método de Muskingum. Ésta es la técnica hidrológica más difundida para manejar relaciones variables de descargasalmacenamientos (Chow et al., 1989). Este método modela el almacenamiento volumétrico de los hidrogramas a lo largo de los cauces de los ríos mediante la combinación de dos almacenamientos conceptuales: uno en forma de cuña y otro en forma de prisma. La ecuación fundamental de este método es:

$$
Q_{j+1}=C_{1} I_{j+1}+C_{2} I_{j}+C_{3} Q_{j}
$$

donde $I_{j}$ es caudal entrante en un tramo en el instante $j ; Q_{j}$ es caudal saliente del mismo tramo en el instante $j$; y $C_{l}$, $C_{2}$ y $C_{3}$ son constantes que dependen de los parámetros $k$ y $x$, que representan un tiempo de circulación o de tránsito, con dimensiones de tiempo, y un coeficiente de almacenamiento adimensional, respectivamente. El valor de $x$ depende de la forma del almacenamiento en cuña modelado, así el valor de $x$ oscila entre 0 , para un almacenamiento sin cuña (embalse), y 0.5 para el caso de que exista una cuña perfectamente definida. El modelo es poco sensible al valor de $x$ y normalmente para corrientes naturales se asume un valor medio próximo a 0.2 (Chow et al., 1989). El tiempo de tránsito o circulación del hidrograma de avenida es $k$ en cada uno de los tramos de cauces de ríos existentes en el modelo.

Las hipótesis de partida o condicionantes de los que se 
parte son las siguientes: la simulación se limita a eventos de lluvia (modelo de eventos) como consecuencia de la propia aplicación del modelo a la simulación de avenidas; la modelación parte de la base de simular únicamente la escorrentía superficial directa, el flujo base se estima de forma previa a la aplicación del modelo; y no se tiene en cuenta la nieve, partimos de eventos en los que no hay nieve.

\section{Implementación del modelo HEC-HMS}

\section{Modelo estructural}

La estructura fisiográfica de la cuenca basada en la red fluvial es el punto de partida de este modelo hidrológico. Para ello hay que establecer, por una parte, las superficies generadoras de hidrogramas de escorrentía (sistemas hidrológicos o subcuencas), y, por otra, las unidades o sistemas hidráulicos por los que circulan dichos hidrogramas (cauces, embalses, etc.).

La información de partida para determinar este modelo estructurado de subcuencas y canales ha sido la red fluvial del mapa cartográfico de Navarra 1:25.000 y el Modelo de Elevación Digital MED (25x25) del Gobierno de Navarra. Se ha trabajado con el Sistema de Información Geográfico SIG ArcView 3.2 de ESRI. A partir de la información tratada y generada en ArcView 3.2 se ha utilizado HECGeoHMS 1.1. para volcar dicha información al HEC-HMS y construir el modelo conceptual de subcuencas y canales. El proceso seguido es el siguiente:

1.- Determinar el contorno de la cuenca objeto de estudio a partir del MED mencionado y considerando el punto definido por la estación de aforo de Arazuri como punto de drenaje de la red fluvial de la cuenca.

2.- Delimitación de las subcuencas atendiendo a distintos criterios:

2.a. Se parte de las subcuencas generadas por la red de afluentes principales: Arga, Ulzama y Elorz.

2.b. Cada una de estas subcuencas se subdivide a su vez en otras de superficie similar, dividiendo los tramos de cauce en tramos de longitud parecida.

2.c. El embalse de Eugui, como tiene una estación de aforo a la salida, se ha considerado como una subcuenca que aporta el hidrograma registrado en la estación de aforo. 2.d. La cuenca vertiente a la balsa de Ezkoriz, al ser muy pequeña y no tener desagüe, se considera como un pozo.

De esta forma se llega a la estructura de subcuencas, mostrada en la Figura 5a, que en HEC-HMS genera el modelo conceptual que se presenta en la Figura $5 \mathrm{~b}$ (izquierda) con la terminología que también se muestra en la misma figura (derecha).

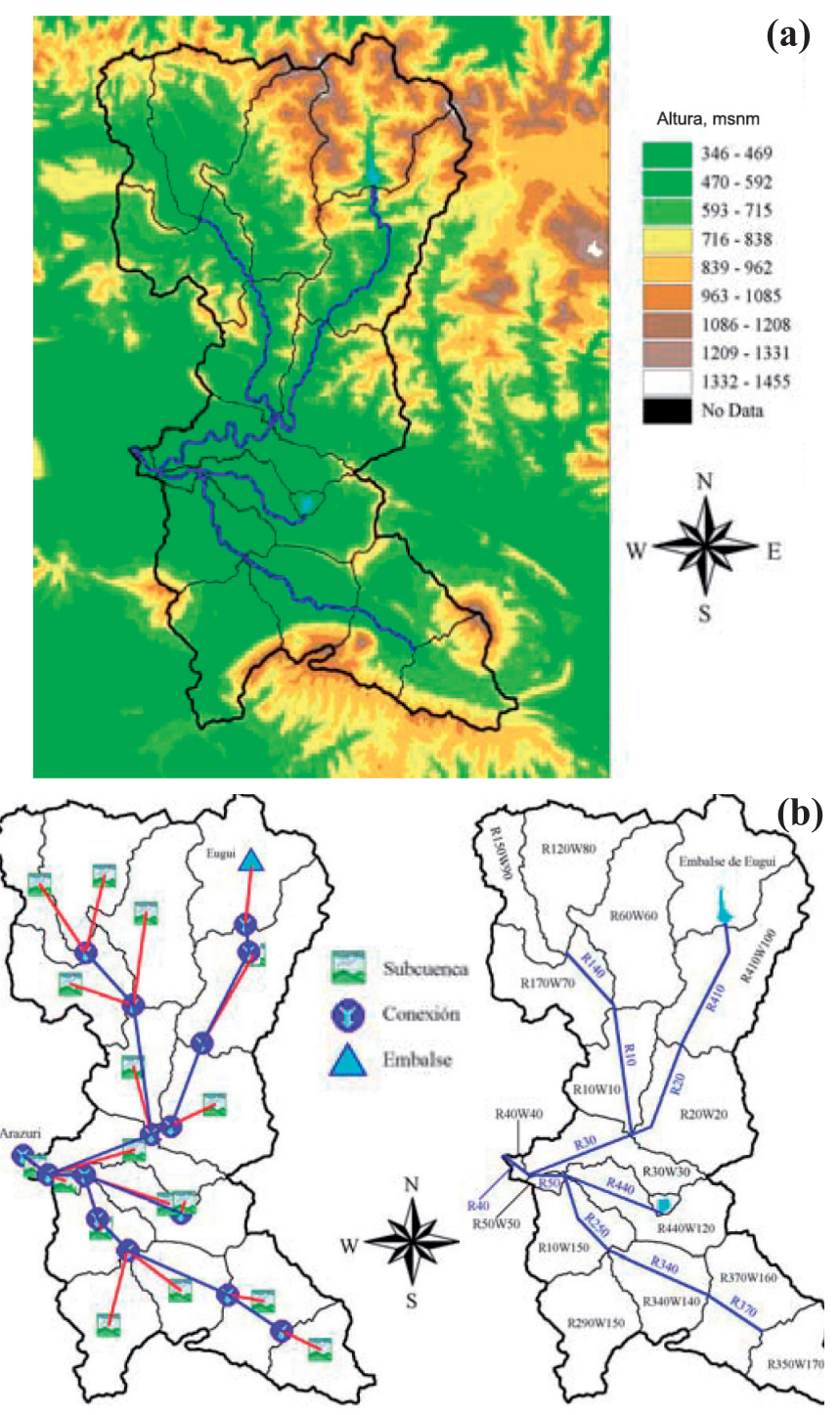

Figura 5: Representación de la cuenca del Arga en Arazuri, con a) la red fluvial y dividida en subcuencas, y b) el modelo conceptual HEC-HMS con los sistemas hidrológicos (subcuencas) e hidráulicos (cauces).

\section{Precipitación espacial}

La entrada al sistema como ya se ha explicado es la precipitación, cuyo valor espacial en cada subcuenca se 

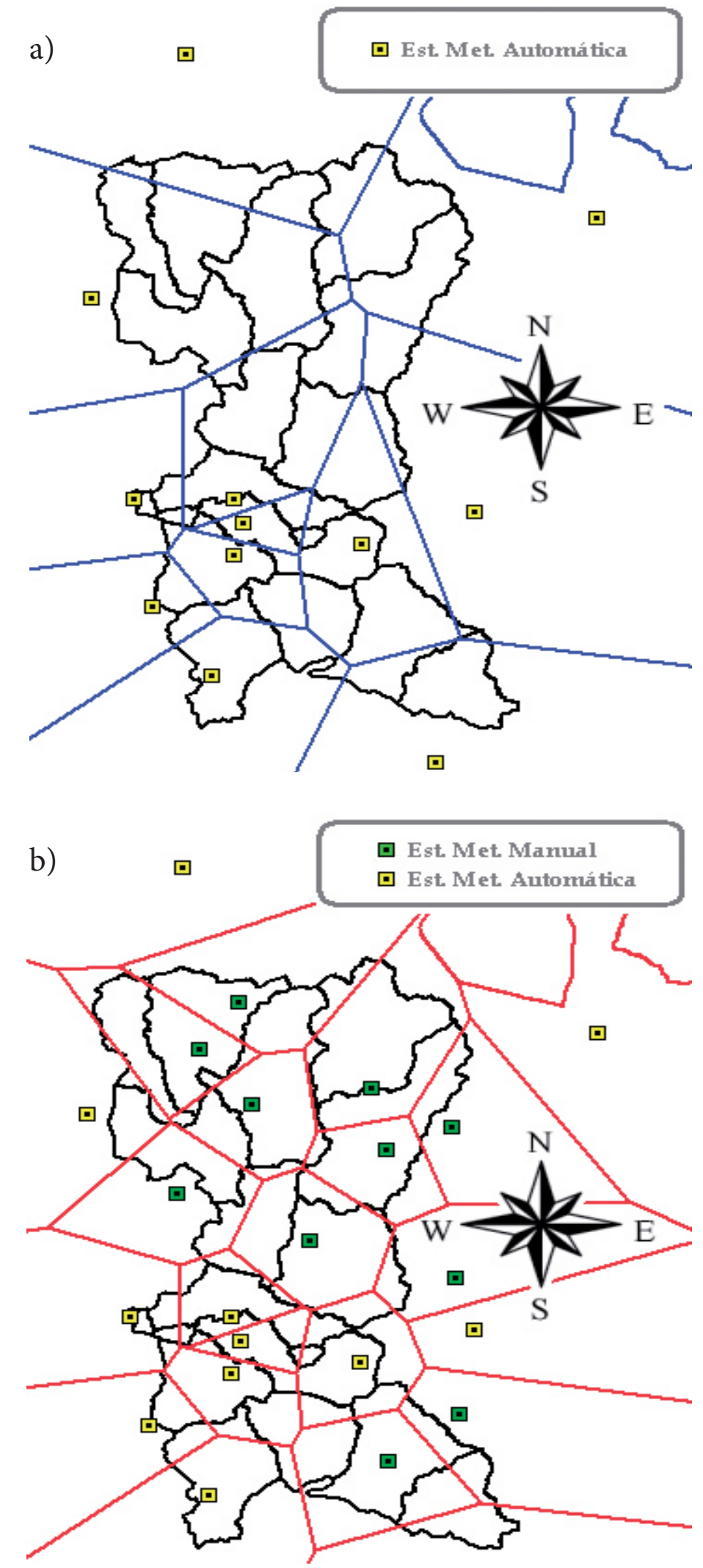

Figura 6: Áreas de influencia determinadas mediante Polígonos de Thiessen: a) únicamente de las estaciones meteorológicas automáticas EMA y b) de ambas automáticas y manuales conjuntamente EMAs + EMMs determina por el método de los polígonos de Thiessen, según la ecuación (1). En la Figura 6a se representan las EMAS con influencia sobre la cuenca objeto de estudio y sus áreas de influencia. Se comprueba que en la zona de cabecera, la más montañosa y pluviosa, existen pocas estaciones, lo que supone un déficit de información importante para la estimación del hietograma de entrada al sistema. Si, por otra parte, se representan las EMM existentes en la cuenca, Figura $6 \mathrm{~b}$, se observa que esa zona queda bastante bien cubierta de estaciones. Por este motivo se ha simulado el modelo bajo estas dos consideraciones: solo con las EMAs y con las EMAs+EMMs, asumiendo la distribución temporal de los valores diarios de estas últimas según la distribución de las automáticas, de forma que el dato de diez minutos para las EMMs se ha estimado según (7).

$$
D D E M M=\sum_{i=1}^{n}\left[\left(D D E M A_{i} \frac{P a_{E M M}}{P a_{E M A n}}\right) C p_{i}\right]
$$

Donde, $D D E M M$ corresponde a dato de 10 minutos de la estación manual, $D D E M A_{i}$ dato de 10 minutos de la estación automática $i, n$ es el número de EMAs con influencia en la estación manual, $P a_{E M M}$ es la precipitación diaria de la $E M M, P a_{E M M}$ es la precipitación acumulada en un día de las $E M A s$ y $C p_{i}$ es el coeficiente de ponderación areal de la $E M A_{i}$.

\section{Caracterización de las subcuencas}

En una subcuenca hay dos procesos que es preciso caracterizar: la determinación de la precipitación efectiva y la circulación de la misma por la cuenca; que en ambos casos se ha elegido el método del SCS. Hay que estimar, por lo tanto, el valor del $C N$ y el de los parámetros asociados a él, $S$ e $I_{a}$, en el primer caso y del $T_{l}$ en el segundo.

Para la estimación del $C N$ en cuencas no aforadas se parte de tablas que han sido elaboradas por diferentes Organismos de la Administración de EE.UU. (SCS, 1993; USDA 1959a, b) disponibles en Ponce (1989). En estas tablas de valores de $C N$ se intentan describir situaciones complejas en las que se combinan distintas condiciones: grupo hidrológico de suelo, tratamiento y uso del suelo, condición hidrológica de la superficie, y condición antecedente de humedad. Con estas tablas y la información 
digital disponible de la cuenca: mapa de suelos 1:50.000, mapa de usos 1:25.000 (Gobierno Navarra, 2001) se ha elaborado el mapa de $C N$ de la cuenca del Arga para unas condiciones de humedad de referencia medias. Con este mapa se han determinado los valores de $C N$ medios de las distintas subcuencas, así como los demás parámetros, $S$ e $I_{a}$, cuyos valores se presentan en la Tabla 1. Aplicando a estos valores medios los criterios de la condición de humedad antecedente establecidos por el método (Ponce, 1989), en función de las zonas de influencia de cada estación meteorológica, se obtienen los valores de $C N$ a aplicar en cada subcuenca y para cada evento.

Tabla 1: Valores de las áreas y de los parámetros hidrológicos medios en cada una de las subcuencas: $\mathrm{CN}, I_{a} \mathrm{y} T_{l}$.

\begin{tabular}{|l|c|c|c|c|}
\hline Subcuenca & $\begin{array}{c}\text { Área } \\
\mathrm{km}^{2}\end{array}$ & $C N$ & $I_{a}, \mathrm{~mm}$ & $T_{1}, \mathrm{~min}$ \\
\hline R10W10 & 43,618 & 69 & 22,82 & 26,40 \\
R20W20 & 67,716 & 69 & 22,82 & 26,04 \\
R30W30 & 39,451 & 85 & 8,96 & 23,15 \\
R40W40 & 2,341 & 85 & 8,96 & 6,30 \\
R50W50 & 3,246 & 85 & 8,96 & 7,32 \\
R60W60 & 74,530 & 62 & 31,14 & 33,30 \\
R170W70 & 52,553 & 65 & 27,35 & 28,50 \\
R120W80 & 61,437 & 64 & 28,58 & 32,28 \\
R150W90 & 32,739 & 66 & 26,17 & 25,80 \\
R410W100 & 83,099 & 62 & 31,14 & 37,38 \\
R440W120 & 45,535 & 80 & 12,70 & 21,96 \\
R310W130 & 41,029 & 81 & 11,92 & 16,20 \\
R340W140 & 40,997 & 78 & 14,33 & 18,06 \\
R290W150 & 50,085 & 76 & 16,04 & 20,22 \\
R370W160 & 56,328 & 73 & 18,79 & 17,04 \\
R350W170 & 37,076 & 70 & 21,77 & 16,08 \\
R390W180 & 68,836 & 61 & 32,48 & 23,34 \\
R420W190 & 2,866 & 81 & 11,92 & 4,50 \\
\hline
\end{tabular}

En lo que se refiere a la caracterización de la escorrentía a lo largo de la cuenca, es decir la generación del hidrograma en la subcuenca, el método utilizado es el HU del SCS, lo que implica que el parámetro a estimar es el tiempo de desfase, $T_{l}$, que se estima mediante la expresión (5). Los valores de este parámetro para cada subcuenca también se presentan en la Tabla 1.

\section{Caracterización de los canales}

En este epígrafe se trata de estimar los parámetros del método de Muskimgum que son el tiempo de circulación, $k$, y el coeficiente de almacenamiento, $x$. Este último parámetro ya se ha indicado que es poco sensible y se adopta el valor de 0.2 (Chow et al., 1989). En el caso del tiempo de tránsito o circulación del hidrograma de avenida, $k$, para cada uno de los tramos de cauces de ríos, se ha estimado a partir de la celeridad de la onda de avenida, cuyo valor se ha determinado en base a estudios hidráulicos, realizados por el Ayuntamiento de Pamplona, y a valores de pendiente medias de los tramos. Cada tramo se ha dividido en subtramos, cuyo número se ha determinado al dividir la longitud de cada tramo por el producto de la celeridad y el intervalo de tiempo de simulación elegido. En la Tabla 2 se presentan los valores de los parámetros del modelo de Muskingum para cada uno de los canales considerados en el modelo de la cuenca representado en la Figura 5.

Tabla 2: Valores de los parámetros del método de Muskingum: $k$ y $x$, y de la celeridad, $c$, para los distintos cauces.

\begin{tabular}{|c|c|c|c|c|c|}
\hline Tramo & Longitud, $\mathrm{m}$ & $c, \mathrm{~m} / \mathrm{s}$ & $x$ & $k, \mathrm{hr}$ & Subtramos \\
\hline $\mathrm{R} 10$ & 14119,3 & 1,5 & 0,2 & 2,6 & 16 \\
$\mathrm{R} 20$ & 4106,7 & 1,1 & 0,2 & 1,0 & 6 \\
R40 & 2894,0 & 1,3 & 0,2 & 0,6 & 4 \\
R30 & 14123,9 & 1,3 & 0,2 & 3,0 & 18 \\
R50 & 3529,9 & 1,5 & 0,2 & 0,7 & 4 \\
R140 & 6447,7 & 1,5 & 0,2 & 1,2 & 7 \\
R200 & 8309,1 & 1,1 & 0,2 & 2,1 & 13 \\
R250 & 4748,1 & 1,1 & 0,2 & 1,2 & 7 \\
R280 & 4437,0 & 1,1 & 0,2 & 1,1 & 7 \\
R280 & 4437,0 & 1,1 & 0,2 & 1,1 & 7 \\
R340 & 15309,8 & 1,3 & 0,2 & 3,3 & 20 \\
R370 & 6241,6 & 1,1 & 0,2 & 1,6 & 9 \\
R380 & 10863,7 & 1,5 & 0,2 & 2,0 & 12 \\
R410 & 3661,0 & 1,5 & 0,2 & 0,7 & 4 \\
R440 & 10742,9 & 1,1 & 0,2 & 2,7 & 16 \\
\hline
\end{tabular}




\section{Descripción de los eventos y extracción del flujo base}

Para el análisis y aplicación del modelo HEC-HMS se han seleccionado 5 eventos de avenida, los más representativos en cuanto a magnitud y efectos en la urbe de los años en los que se dispone de datos de precipitación cada diez minutos, es decir, de las estaciones meteorológicas automáticas. ¿Por qué solo de estos años?, porque es de los que se dispone de la distribución temporal de la lluvia. Las características de estos eventos se presentan en la Figura 7 y en la Tabla 3.

Como ya se ha mencionado anteriormente, en este estudio utilizamos el HEC-HMS considerando únicamente la componente de flujo superficial directo. Es preciso, por lo tanto, estimar el flujo base y separarlo del hidrograma de caudal observado en la estación de aforo, para poder compararlo con el simulado por el modelo. La extracción del flujo base se ha realizado utilizando un filtro recursivo, concretamente, el propuesto por Eckhardt (2005) de dos parámetros. Su expresión viene dada por la ecuación (8):

$q_{i}=\frac{\left(1-B F I_{\max }\right)}{1-a B F I_{\max }}\left[Q_{i}-a\left(Q_{i-1}-q_{i-1}\right)\right] ; \quad q_{i} \geq 0$

donde $q_{i}$ es la escorrentía directa en el instante $i, Q_{i}$ es la escorrentía total en el instante $i, a$ es una constante de recesión del flujo subterráneo y $\mathrm{BFI}_{\max }$ es el índice del flujo subterráneo. El parámetro $a$, se relaciona con la recesión del flujo base mientras que el parámetro $B F I_{\max }$, indica la máxima proporción de flujo base, con respecto a la escorrentía total, que se alcanzaría en la condición de equilibrio, si el almacenamiento de la cuenca se mantuviese constante en un periodo de tiempo suficientemente largo.

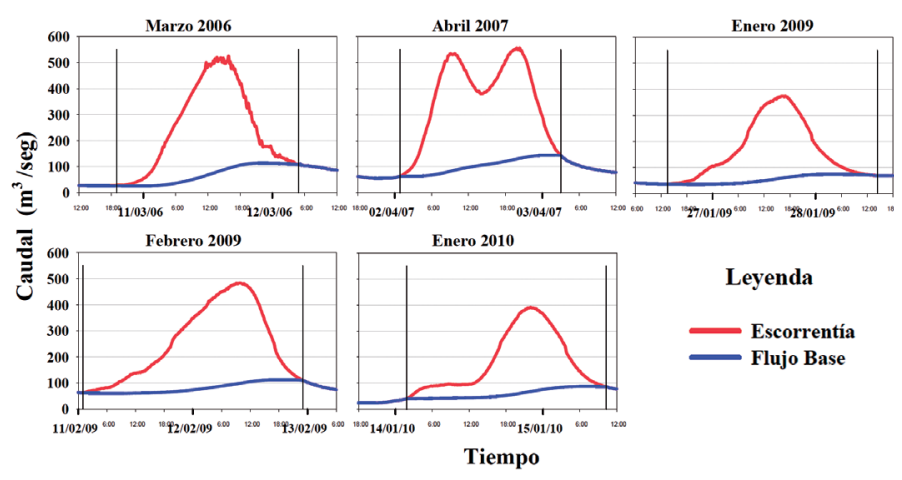

Figura 7: Representación de los hidrogramas observados de cada evento en la estación de Arazuri con la separación del flujo base
Tabla 3: Valores de las características de cada evento y de los parámetros del filtro de Eckhardt (2005) a y $B F I_{\max }$

\begin{tabular}{|c|c|c|c|c|}
\hline EVENTO & $\begin{array}{c}\text { Duración } \\
\text { días }\end{array}$ & $Q_{\max } \mathrm{m}^{3 / \mathrm{s}}$ & $a$ & $B F_{\max }$ \\
\hline Marzo 2006 & 10 al 12 & 525.5 & 0,998 & 0,6 \\
Abril 2007 & 2 al 4 & 556.8 & 0,999 & 0,7 \\
Enero 2009 & 26 al 29 & 374.1 & 0,999 & 0,6 \\
Febrero 2009 & 11 al 13 & 483.9 & 0,999 & 0,6 \\
Enero 2010 & 14 al 15 & 391.6 & 0,999 & 0,7 \\
\hline
\end{tabular}

Los valores de los parámetros se han establecido adoptando criterios gráficos de ajuste, de forma que el hidrograma de escorrentía subsuperficial generado se ajuste adecuadamente al hidrograma observado. Así, el valor del parámetro $a$ se ajustó a un valor de 0.999 y el parámetro $B F I_{\max }$ oscila entre 0.6 y 0.7 . En la Tabla 3 se presentan los valores de dichos parámetros ajustados para cada uno de los eventos.

\section{Análisis y resultados del modelo Análisis de sensibilidad}

El análisis de sensibilidad es fundamental para comprobar el grado de dependencia de los resultados del modelo con respecto a los parámetros más importantes. Se ha realizado sobre los parámetros más significativos de los métodos utilizados: número de curva $C N$; pérdidas iniciales $I_{a}$; tiempo de tránsito de la circulación en cauces $k$ y tiempo de desfase $T_{i}$; y en un hidrograma observado, la avenida de marzo 2006. Para evaluar el grado de sensibilidad se ha determinado el índice de sensibilidad, $I S$, descrito por Nearing et al. (1989):

$$
I S=\left|\frac{\left(O_{\text {Max }}-O_{\text {Min }}\right) / O_{M e d}}{\left(I_{\text {Max }}-I_{\text {Min }}\right) / I_{M e d}}\right|
$$

En donde $I_{M a x}$ es el valor máximo del parámetro a analizar; $I_{\text {Min }}$ es el valor mínimo de dicho parámetro; y $I_{\text {Med }}$ es el valor medio; $O_{\text {Máx }}$ es el valor máximo de la variable de salida a evaluar; $O_{\text {Min }}$ es su valor mínimo; y $O_{\text {Med }}$ es el valor medio. En este caso la variable evaluada ha sido el caudal máximo, 
$Q_{\max }$. Un valor de $I S$ igual a 1 indica que los incrementos de ambas distribuciones de valores son iguales.

El análisis de sensibilidad del parámetro $C N$ se ha realizado utilizando intervalos de valores de los números de curva medios de toda la cuenca. Estos intervalos se han establecido de forma porcentual a la diferencia entre 100 (valor máximo) y el valor del $C N$ medio de la cuenca.

Según el método del $S C S$, el valor de las pérdidas iniciales $I_{a}$, viene dado por el $20 \%$ de las pérdidas potenciales máximas $\left(I_{a}=0.2 \mathrm{~S}\right)$, aunque realmente dicho valor de 0.2 se puede considerar como un coeficiente de pérdidas incierto $C_{p}$, de forma que $I_{a}=C_{p} \mathrm{~S}$. Dado que cuando se trata de avenidas extraordinarias las condiciones iniciales del suelo son críticas, es importante comprobar la sensibilidad del modelo con respecto a la variación del parámetro $C_{p}$, tomando también valores de 0.05 y 0.1 . Otro parámetro importante en la circulación de avenidas es el tiempo de propagación, $k$. Igual que en los casos anteriores, dejando todos los demás parámetros fijos, se ha ejecutado el modelo para valores de $k$ superiores en media hora a los estimados para cada tramo, e inferiores en media hora. En el caso del tiempo de desfase, $T_{l}$, también se ha ejecutado el modelo variando el valor de dicha variable de forma porcentual, tal como se muestra en la Figura 8 y Tabla 4. Los resultados de dicho análisis se presentan en la Figura 8 y en las Tablas 4 y 5 .

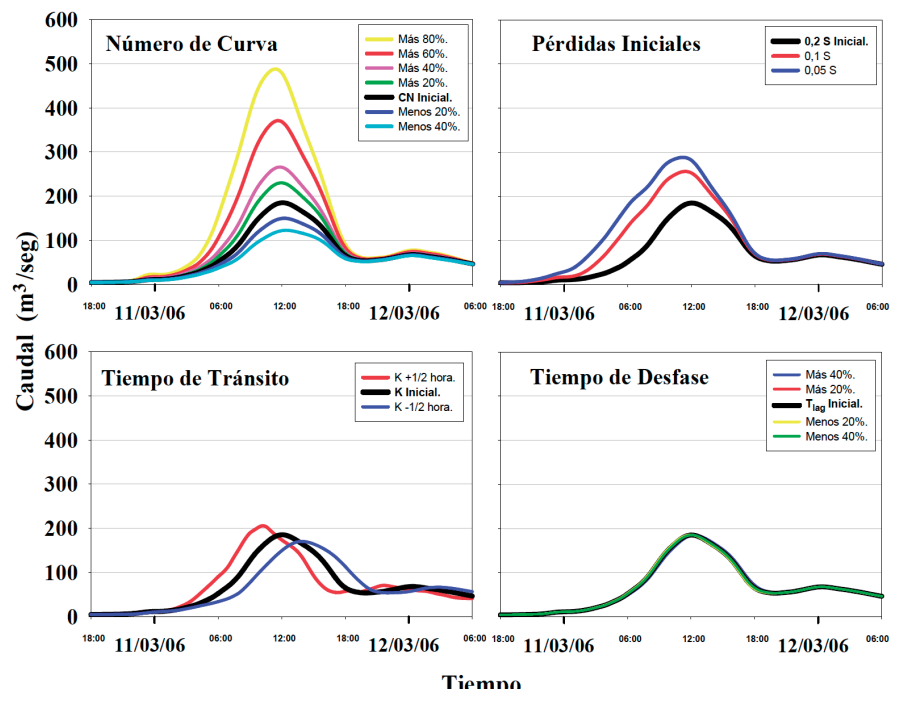

Figura 8: Representación del análisis de sensibilidad de los parámetros más representativos del modelo: $C N, I_{a}, T_{l}, k$
Tabla 4: Valores del tiempo en el máximo $T_{p}$, y del caudal máximo $\mathrm{Q}_{\max }$, estimados para los distintos escenarios del análisis de sensibilidad considerados

\begin{tabular}{|c|c|c|c|c|}
\hline Parámetro & Variación & $\begin{array}{l}\text { Valor pa- } \\
\text { rámetro }\end{array}$ & $T_{p}, \mathrm{hr}$ & $Q_{p}, \mathrm{~m}^{3} / \mathrm{s}$ \\
\hline \multirow{7}{*}{$C N$} & $C N_{\text {ref }}-40 \%$ & 57 & $12: 30$ & 122,5 \\
\hline & $C N_{\text {ref }}-20 \%$ & 63 & $12: 00$ & 149,5 \\
\hline & $C N_{\text {ref }}$ & 71 & $12: 00$ & 185,1 \\
\hline & $C N_{\text {ref }}+20 \%$ & 76 & $12: 00$ & 230,1 \\
\hline & $C N_{\text {ref }}+40 \%$ & 82 & $12: 00$ & 265,3 \\
\hline & $C N_{\text {ref }}+60 \%$ & 88 & $11: 30$ & 370,8 \\
\hline & $C N_{\text {ref }}+80 \%$ & 94 & $11: 30$ & 487,7 \\
\hline \multirow{3}{*}{$I_{a}, \mathrm{~mm}$} & $I_{a_{\mathrm{ref}}}=0,20 \mathrm{~S}$ & 22,9 & $12: 00$ & 185,1 \\
\hline & $I_{a_{\mathrm{ref}}}=0,10 \mathrm{~S}$ & 11,5 & $11: 30$ & 256,6 \\
\hline & $I_{a_{\text {ref }}}=0,05 S$ & 5,7 & $11: 00$ & 288,3 \\
\hline \multirow{3}{*}{$k, \mathrm{hr}$} & $k_{\mathrm{ref}}-0,5$ & 1,2 & $10: 00$ & 204,3 \\
\hline & $k_{\text {ref }}$ & 1,7 & $12: 00$ & 185,1 \\
\hline & $k_{\text {ref }}+0,5$ & 2,2 & $14: 00$ & 170,1 \\
\hline \multirow{3}{*}{$T_{l}, \mathrm{hr}$} & $T_{\text {lref }}$ & 21,3 & $12: 00$ & 185,1 \\
\hline & $T_{\text {lref }}+20 \%$ & 25,6 & $12: 00$ & 184,5 \\
\hline & $T_{\text {lref }}+40 \%$ & 29,9 & $12: 30$ & 184,8 \\
\hline
\end{tabular}

De la Figura 8 y de la Tabla 4, se deduce que el número de curva $C N$, es el parámetro más sensible, su variación tiene un gran efecto sobre el valor del caudal simulado (hidrograma), fundamentalmente con respecto al volumen del hidrograma y al caudal máximo o punta y, sin embargo, es menos sensible respecto al tiempo en el máximo (Figura 8). El parámetro de pérdidas iniciales $I_{a}$, también manifiesta un efecto importante sobre el volumen de escorrentía.

Una disminución del valor de $I_{a}$ hace que el aporte de la lluvia caída se trasforme con mayor rapidez en escorrentía, anticipando y elevando la curva de ascenso del hidrograma. Consecuencia de esto es que el caudal máximo $\mathrm{Q}_{\max }, \mathrm{y}$ el tiempo en el máximo $T_{p}$, sean sensibles al valor $I_{a}$, aunque 
mucho menos al segundo que al primero. En referencia al tiempo de tránsito $k$, es evidente que la disminución o el aumento del valor de dicho parámetro provocará una anticipación o un retardo respectivamente en el hidrograma de salida y, por lo tanto, del tiempo en el máximo $T_{p} \mathrm{y}$, por lo tanto, un aumento o disminución respectivamente del valor del caudal máximo $\mathrm{Q}_{\max }$. Por último, en la Figura 8 se pone de manifiesto que el efecto de la variación del tiempo de desfase $T_{l}$, en el hidrograma simulado es inapreciable, mucho menor que el de los otros parámetros analizados.

Tabla 5 : Valores del índice de Nearing IS, obtenidos por intervalos de valores de los parámetros $C N, k$, y $I_{a}$

\begin{tabular}{|l|l|l|}
\hline Parámetros & Intervalos de variación & I. Nearing IS \\
\hline \multirow{5}{*}{$C N$} & $C N_{\text {ref }}-40 \%$ y $C N_{\text {ref }}-20 \%$ & 1,99 \\
& $C N_{\text {ref }}-20 \%$ y $C N_{\text {ref }}$ & 1,78 \\
& $C N_{\text {ref }}$ y $C N_{\text {ref }}+20 \%$ & 3,19 \\
& $C N_{\text {ref }}+20 \%$ y $C N_{\text {ref }}+40 \%$ & 1,87 \\
& $C N_{\text {ref }}+40 \%$ y $C N_{\text {ref }}+60 \%$ & 4,70 \\
& $C N_{\text {ref }}+60 \%$ y $C N_{\text {ref }}+80 \%$ & 4,13 \\
\hline \multirow{2}{*}{$I, \mathrm{~mm}$} & $0,20 S$ y $0,10 S$ & 2,06 \\
& $0,10 S$ y $0,05 S$ & 4,28 \\
\hline \multirow{2}{*}{$k, \mathrm{hr}$} & $k_{\text {ref }}$ y $k_{\text {ref }}-0,5$ & 3,45 \\
& $k_{\text {ref }}$ y $k_{\text {ref }}+0,5$ & 3,08 \\
\hline
\end{tabular}

Para cuantificar este efecto o sensibilidad de los parámetros, se ha utilizado el índice de sensibilidad de Nearing, IS, ecuación (9). El valor de este índice se ha estimado por intervalos del valor del parámetro, para ver también como varía la sensibilidad en función de los valores.

De esta forma, se ha podido apreciar que el modelo es muy sensible al valor de $C N$ fundamentalmente para valores elevados, así mismo se ha comprobado que el modelo también es muy sensible al valor de $I_{a}$, pero en este caso la sensibilidad es mayor para valores bajos del parámetro. En el caso del parámetro $k$, se ha observado que su sensibilidad no depende de la magnitud de su valor. Y en el caso del $T_{l}$, el modelo es tan insensible a su valor, valores del $I S$ próximos a cero, que se han omitido los resultados.

\section{Resultados}

Como se ha comentado en la implementación del modelo, debido a la escasez EMAs en la cuenca, se han realizado dos simulaciones: considerando únicamente EMAs; y utilizando ambas, automáticas EMAs y manuales EMMs. En la Figura 9 se observa como al tener en cuenta las EMMs mejora el resultado de la simulación, evidentemente la mejora no es uniforme dependerá de la distribución espacial y temporal de la lluvia. No obstante, de este análisis gráfico preliminar ya se concluye que el resultado de la simulación en el caso de EMAs+EMMs es bastante aceptable para ser una mera aplicación del modelo HECHMS sin ningún tipo de calibración, a excepción del evento de abril de 2007 que es un episodio compuesto con dos puntas. En estos episodios complejos, la incertidumbre en la estimación del flujo base es mayor, y esa incertidumbre es transmitida a las hipótesis de partida de la metodología seleccionada para determinar el hidrograma de escorrentía directa de salida de las cuencas.

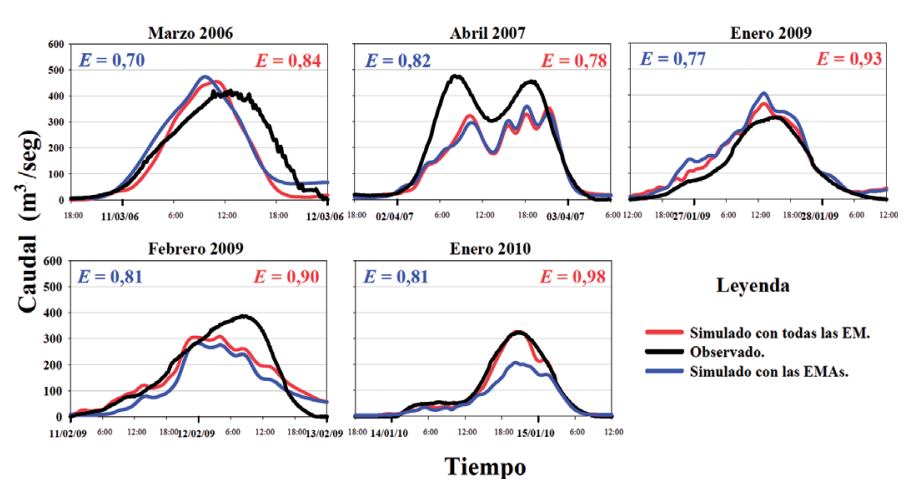

Figura 9: Comparación de los resultados obtenidos mediante la simulación con EMAs y con EMAs + EMMs

Para evaluar estos resultados de la aplicación del modelo se han determinado los errores absolutos y los relativos en $\%$ de diferentes parámetros, como el volumen del hidrograma, $V E D$, el caudal máximo o punta $Q_{\max }$, y el tiempo en el máximo, $T_{p}$. También se ha calculado el valor de la Eficiencia $E$, según Nash y Sutcliffe (1970) dado por la ecuación (10), que refiere el grado de error obtenido de forma normalizada y adimensional.

$$
E=1-\frac{\sum_{j=1}^{m}\left[Q_{o b s, j}-Q_{s i m, j}\right]^{2}}{\sum_{j=1}^{m}\left[Q_{o b s, j}-\bar{Q}_{o b s}\right]^{2}}
$$


Tabla 6: Valores de los errores relativos y de la Eficiencia $E$, obtenidos en la estimación de $Q_{\max }$, $T_{p}$ y en el Volumen de Escorrentía Directa $V E D$

\begin{tabular}{|c|c|c|c|c|c|c|}
\hline \multirow{2}{*}{\multicolumn{2}{|c|}{ Parámetros }} & \multicolumn{5}{|c|}{ Eventos } \\
\hline & & \multirow{2}{*}{$\begin{array}{c}\text { Marzo } \\
2006 \\
419,40 \\
\end{array}$} & \multirow{2}{*}{$\begin{array}{c}\text { Abril } \\
2007 \\
475,60\end{array}$} & \multirow{2}{*}{$\begin{array}{c}\begin{array}{c}\text { Enero } \\
2009\end{array} \\
387,20 \\
\end{array}$} & \multirow{2}{*}{$\begin{array}{c}\text { Febrero } \\
2009 \\
314,80\end{array}$} & \multirow{2}{*}{$\begin{array}{c}\begin{array}{c}\text { Enero } \\
2010\end{array} \\
324,00 \\
\end{array}$} \\
\hline \multirow{4}{*}{$\begin{array}{l}\text { Caudal } \\
\text { máximo } \\
Q_{\max }, \mathrm{m}^{3} / \mathrm{s}\end{array}$} & Observado & & & & & \\
\hline & Simulado & 455,50 & 345,60 & 321,00 & 358,90 & 322,00 \\
\hline & Dif. abs & $-36,10$ & 130,00 & 66,20 & $-44,10$ & 2,00 \\
\hline & Dif. (\%) & $-8,61$ & 27,33 & 17,10 & $-14,01$ & 0,62 \\
\hline \multirow{4}{*}{$\begin{array}{l}\text { Tiempo en } \\
\text { punta } T_{p}, \mathrm{hr}\end{array}$} & Observado & 35,83 & 27,00 & 8,00 & 26,67 & 20,00 \\
\hline & Simulado & 19,83 & 38,83 & 7,50 & 27,50 & 29,00 \\
\hline & Dif. Abs. & 16,00 & $-11,83$ & 0,50 & $-0,83$ & $-9,00$ \\
\hline & Dif. (\%) & 44,66 & $-43,81$ & 6,25 & $-3,11$ & $-45,00$ \\
\hline \multirow{4}{*}{$\begin{array}{l}\text { Volumen } \\
\text { de VED, } \\
\mathrm{m}^{3}\end{array}$} & Observado & 20.727 .060 & 28.457 .280 & 20.169 .540 & 28.738 .920 & 14.714 .880 \\
\hline & Simulado & 17.282 .160 & 18.572 .400 & 22.879 .800 & 30.086 .040 & 13.191 .900 \\
\hline & Dif. & 3.444 .900 & 9.884 .880 & -2.710 .260 & -1.348 .120 & 1.522 .980 \\
\hline & Dif. (\%) & 16,62 & 34,74 & $-13,44$ & $-4,69$ & 10,35 \\
\hline \multicolumn{2}{|l|}{ Eficiencia $E$} & 0,84 & 0,78 & 0,93 & 0,90 & 0,98 \\
\hline
\end{tabular}

Donde $Q_{\text {sim }}$ es el caudal simulado; $Q_{o b s}$ es el caudal observado y $\bar{Q}_{o b s}$ es el caudal medio observado. Este índice representa la relación entre la varianza del error y la varianza de la serie de observaciones, valores cercanos a 1 implican un buen ajuste y valores menores o cercanos a 0 , indican que el modelo no es mejor que usar el promedio de las observaciones.

Todos estos índices de ajuste se presentan en la Tabla 6 para cada evento. Se pone de manifiesto de nuevo lo ya comentado, es decir, la bondad de los ajustes en los resultados excepto para el evento de abril del 2007. En los valores de eficiencia, hay tres eventos por encima de 0.9 y los otros dos están o próximos a 0.8 o por encima. Las diferencias relativas respecto al caudal punta y al volumen de escorrentía son bastante aceptables, excepto el caso indicado.

\section{Conclusiones}

Los buenos resultados obtenidos en la aplicación del modelo HEC-HMS en la cuenca del río Arga a su paso por Pamplona, a los 5 sucesos de avenida más significativos de los últimos 10 años, dan por válido el trabajo previo de caracterización física e hidrológica de la cuenca, como la estructura fisiográfica de la cuenca y la asignación de valores a los distintos parámetros $C N, T_{b} x, k$, que caracterizan los procesos hidrológicos considerados. Únicamente hay que indicar que la densidad o el número de estaciones meteorológicas automáticas, que son las que permiten registrar la distribución temporal de las tormentas, es escaso en la zona de cabecera de la cuenca, precisamente en la zona más montañosa y con más pluviometría, lo que a efectos de valoración de recursos hídricos es un déficit importante.

Del análisis de sensibilidad, se concluye que el número de curva $C N$, es el parámetro más sensible, su variación tiene un gran efecto sobre el valor del caudal simulado (hidrograma).

Con respecto a las pérdidas iniciales $I_{a}$, se aprecia también 
que es bastante sensible aunque en menor medida, su variación provoca un efecto importante en el volumen de escorrentía. En referencia al tiempo de tránsito $k$, es evidente que la disminución o el aumento del valor de dicho parámetro provocará una anticipación o un retardo respectivamente en el hidrograma de salida $\mathrm{y}$, por lo tanto, del tiempo en el máximo $T_{p}$; así como un aumento o disminución respectivamente del valor del caudal máximo $Q_{\max }$. Por último, el tiempo de desfase $T_{l}$, es un parámetro poco sensible en el hidrograma simulado, mucho menor que el de los otros parámetros analizados.

La introducción de los datos de las series de precipitación diaria, con las distribuciones temporales ponderadas de las series de precipitación de las estaciones automáticas (cada 10 minutos), mejoran significativamente los resultados de la simulación, es decir, los hidrogramas simulados.

Los resultados de la aplicación del modelo, aportan valores de eficiencia $E$, muy satisfactorios en cuatro de cinco avenidas; $y$, en general, aportan mejores resultados para los caudales, caudal punta y volumen de escorrentía, que para el tiempo en el máximo.

\section{Agradecimientos}

Se quiere expresar el agradecimiento tanto al Servicio de Aguas del Gobierno de Navarra como a Sección de Hidrología de la Confederación Hidrológica del Ebro por haber facilitado todo tipo de información para llevar a cabo este trabajo.

\section{Referencias}

Al-Abed, N., Abdulla, F. and Abu Khyarah, A. (2005). GIShydrological models for managing water resources in the Zarqa River basin. Environmental Geology 47(3), 405-411.

Ayuntamiento de Pamplona (2003). Plan Municipal de Pamplona. Boletín Oficial de Navarra (BON) 02/05/2003.

Bedient, P.B. and Huber, W.C. (1990). Hydrology and Floodplain Analysis. Addison-Wesley, Reading

CEE (2007). Directiva 2007/60/CE del Parlamento Europeo y del Consejo, del 23 de octubre del 2007, relativa a la evaluación y gestión de los riesgos de inundación. Diario Oficial de la Unión Europea (06/11/2007), L: 288: 27-34.

Chow, V.T., Maidment, D.R. and Mays, L.W. (1989). Applied Hydrology. McGraw-Hill
Chu, X. and Steinman, A. (2009). Event and continuous hydrologic modeling with HEC-HMS. Journal of Irrigation and Drainage Engineering 135(1), 119-124.

Cunderlik, J. and Simonovic, S.P. (2004). Calibration, verification and sensitivity analysis of the HEC-HMS hydrologic model. Water Resources Research Report. Book 11. Department of Civil and Environmental Engineering, University of Western Ontario. http://ir.lib.uwo.ca/wrrr/11.

Cydzik, K. and Hogue, T.S. (2009). Modeling postfire response and recovery using the Hydrologic Engineering Center Hydrologic Modeling System (HEC-HMS). Journal of the American Water Resources Association 45( 3), 702-714.

Eckhardt, K. (2005). How to construct recursive digital filters for baseflow separation. Hydrological Processes 19, 507-515

Emerson, C.H., Welty, C. and Traver R.G. (2005). Watershedscale evaluation of a system of storm water detention basins. Journal of Hydrologic Engineering 10(3), 237-242

Gobierno de Navarra (2001). Mapa de Cultivos y Aprovechamientos de Navarra (MCAN) 1:25.000. Departamento de Agricultura, Ganadería y Alimentación del Gobierno de Navarra. Sección de Suelos y Climatología. Pamplona, Navarra.

Iglesias, D. (2008). Estudio de los sucesos extraordinarios de lluvia y sus efectos en la cuenca del río Arga. Trabajo Final de Carrera. E.T.S.I.A., Universidad Pública de Navarra.

Kang, B. and Ramírez, J.A. (2007). Response of streamflow to weather variability under climate change in the Colorado Rockies. Journal of Hydrologic Engineering 12(1), 63-73.

Knebl, M.R., Yang, Z.L., Hutchison, K. and Maidment, D.R. (2005). Regional scale flood modeling using NEXRAD rainfall, GIS, and HEC-HMS/RAS: a case study for the San Antonio River Basin Summer 2002 storm event. Journal of Environmental Management 75(4), 325-336.

McColl, C. and Aggett, G. (2007). Land-use forecasting and hydrologic model integration for improved land-use decision support. Journal of Environmental Management 84, 494-512.

McLin, S.G., Springer, E.P. and Lane, L.J. (2001). Predicting floodplain boundary changes following the Cerro Grande wildfire. Hydrological Processes 15(15), 2.967-2.980.

Mockus, V. (1969). National Engineering Handbook, section 4: Hydrology. United States Department of Agriculture (USDA). Soil Conservation Service (SCS), Washington, D.C.

Nash, J.E. and Sutcliffe, J.V. (1970). River flow forecasting through conceptual models I: A discussion of principles. Journal of Hydrology 10, 282-290 
Nearing, M.A., L.D. Ascough, and Chaves H.M.L. (1989). WEPP model sensitivity analysis. WEPP-USDA Water Erosion Prediction Project. USDA-ARS-NSERL, 2, 14.1-14.33.

Ponce, V.M. (1989). Engineering Hydrology: Principles and Practices. Prentice Hall. New Jersey

SCS (1993). Soil Survey Manual. Handbook 18. Soil Survey Division Staff. Agricultural Research Administration. United States Department of Agriculture (USDA).

Treviño, A. y Ortiz, S. (2004). Metodología para la modelación distribuida de la escorrentía superficial y la delimitación de zonas inundables en ramblas y ríos-rambla mediterráneos. Investigaciones Geográficas 35, 67-83.
USDA (1959a). Forest and Range Hydrology Handbook. United States Department of Agriculture, Forest Service. Washington D.C.

USDA (1959b). Handbook on Methods of Hydrology Analysis. United States Department of Agriculture, Forest Service. Washington D.C.

Viessman, W. and Lewis, G.L. (1996). Introduction to Hydrology. Harper Collins. New York.

Yusop, Z., Chan, C.H. and Katimon, A. (2007). Runoff characteristics and application of HEC-HMS for modelling stormflow hydrograph in an oil palm catchment. Water Science \& Technology 56(8), 41-48. 